Old Dominion University ODU Digital Commons

Electrical \& Computer Engineering Faculty

Publications

Electrical \& Computer Engineering

2003

\title{
Electron Bernstein Wave-X-O Mode Conversion and Electron Cyclotron Emission In MAST
}

Josef Preinhaelter

Pavol Pavlo

Vladimir Shevchenko

Martin Valovic

Linda L. Vahala

Old Dominion University, lvahala@odu.edu

See next page for additional authors

Follow this and additional works at: https://digitalcommons.odu.edu/ece_fac_pubs

Part of the Electrical and Computer Engineering Commons, and the Plasma and Beam Physics Commons

\section{Repository Citation}

Preinhaelter, Josef; Pavlo, Pavol; Shevchenko, Vladimir; Valovic, Martin; Vahala, Linda L.; and Vahala, George, "Electron Bernstein Wave-X-O Mode Conversion and Electron Cyclotron Emission In MAST" (2003). Electrical \& Computer Engineering Faculty Publications. 32.

https://digitalcommons.odu.edu/ece_fac_pubs/32

\section{Original Publication Citation}

Preinhaelter, J., Pavlo, P., Shevchenko, V., Valovic, M., Vahala, L., Vahala, G., \& Team, M. (2003). Electron Bernstein wave-X-O mode conversion and electron cyclotron emission in MAST. Review of Scientific Instruments, 74(3), 1437-1440. doi: 10.1063/1.1530383 
Authors

Josef Preinhaelter, Pavol Pavlo, Vladimir Shevchenko, Martin Valovic, Linda L. Vahala, and George Vahala 


\title{
Electron Bernstein wave-X-O mode conversion and electron cyclotron emission in MAST
}

\author{
Josef Preinhaelter and Pavol Pavlo \\ EURATOM/IPP.CR Association, Institute of Plasma Physics, 18221 Prague, Czech Republic \\ Vladimir Shevchenko, Martin Valovic, and MAST Team \\ EURATOM/United Kingdom Atomic Energy Authority Fusion Association, Culham Science Centre, \\ Abingdon OX14 3DB, United Kingdom \\ Linda Vahala ${ }^{a)}$ \\ Department of Electrical \& Computer Engineering, Old Dominion University, Norfolk, Virginia 23529 \\ George Vahala \\ Department of Physics, College of William \& Mary, Williamsburg, Virginia 23185
}

(Presented on 8 July 2002)

\begin{abstract}
Electron cyclotron emission (ECE) from overdense plasmas can only occur due to electron Bernstein waves (EBW) mode converting near the upper hybrid region to an electromagnetic wave. Experimental data of ECE observations on MAST are studied and compared with EBW-X-O mode conversion modeling results. (c) 2003 American Institute of Physics.
\end{abstract}

[DOI: $10.1063 / 1.1530383]$

\section{INTRODUCTION}

In overdense plasmas, like MAST, it is not possible to have radiation of $\mathrm{O}$ or $\mathrm{X}$ modes from regions of the first five electron cyclotron harmonics. However, since electron Bernstein waves (EBW) are not subject to such a density limit, EBW radiation can be detected. In this case, the electrostatic EBW mode converts to an electromagnetic wave near the upper hybrid region (UHR), which can then be measured experimentally. For MAST, such electron cyclotron emission (ECE) data exists from 16 to $75 \mathrm{GHz}$. Here, we interpret the ECE data as resulting from EBW-X-O, a mode conversion process $^{1}$ that has been measured on the W-7 stellarator. ${ }^{2}$

\section{WAVE PROPAGATION MODEL}

To examine the experimental data from MAST, the instantaneous B field and its spatial derivatives are reconstructed from a two-dimensional splining of a toroidally symmetric EFIT equilibrium. The plasma density and temperature profiles in the MAST plasma are obtained from high spatial resolution Thomson scattering measurements.

The spot position at which the antenna is aimed (as well as the $\mathbf{k}$ of the outgoing waves) is determined from the intersection of the antenna pattern with the separatrix (Fig. 1). Only linearly polarized waves are detected, with the plane of polarization selected by the polarization rotator. ${ }^{3}$ For EBW$\mathrm{X}-\mathrm{O}$, the ECE antenna is oriented so that at the plasma boundary the $\mathbf{k}$ and $\mathbf{E}$ field of the obliquely propagating $\mathrm{O}$ mode lie in the plane spanned by the edge density gradient and $\mathbf{B}$ field. Also shown in Fig. 1 is the auxiliary planestratified plasma slab geometry used in the numerical fullwave propagation solution. A schematic of wave propagation

${ }^{\text {a)} E l e c t r o n i c ~ m a i l: ~ 1 v a h a l a @ o d u . e d u ~}$ in MAST is shown in Fig. 2. The O- and X-mode cutoffs and resonances are determined from a cold plasma slab WentzelKramers-Brillouin approximation. We see that the $\mathrm{O}$ and $\mathrm{X}$ modes can propagate only near the plasma boundary, penetrating only slightly into the transport barrier, which was formed in this shot during the H-mode phase. Since this region is only several centimeters thick, a cold plasma slab model can be used for the full-wave solution of the $\mathrm{X}-\mathrm{O}$ mode conversion in the plasma resonance region ${ }^{1,2}$ For low frequencies, the efficiency of $\mathrm{X}-\mathrm{O}$ conversion is practically $100 \%$. For high frequencies, the mode conversion efficiency is reduced as the width of the evanescent region increases and becomes comparable to the wavelength. Thus, the linearly polarized component of the emitted power received by the antenna decreases with frequency (Fig. 3), where the incident $\mathrm{X}$-mode power at the UHR is assumed to be equal to unity. Since the mode-converted oblique $\mathrm{O}$ mode is weakly elliptically polarized, the intensity of the detected linearly polarized component must be less than $0.5-0.7$ of the total O-mode intensity.

In the central plasma only EBW can propagate, and this wave is fully mode-converted to the $\mathrm{X}$ mode at the UHR. Hence, we only need to determine the intensity of the EBW emission from the electron cyclotron regions. Assuming the plasma is in thermal equilibrium in these central regions, the intensity of the blackbody radiation (Rayleigh-Jeans law) $I_{\mathrm{EBW}} \approx \omega^{2} T_{e}$. Thus, we must determine for each $f$ the position at which EBW is absorbed. EBW ray-tracing gives the most reliable results, and work is under way to generalize our lower hybrid code ${ }^{4}$ to handle EBW. These results will be presented elsewhere. Here, we investigate the propagation of EBW in the plasma slab (Fig. 1) using the electrostatic dispersion relation ${ }^{5}$ 


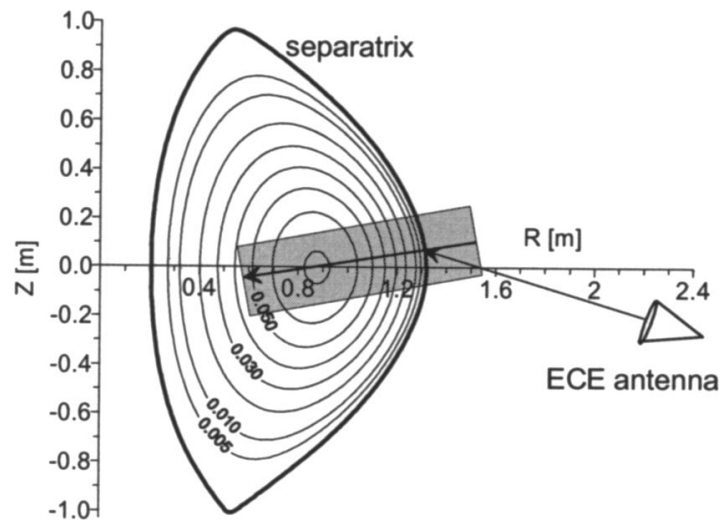

FIG. 1. The RZ-cross section of the antenna geometry orientation. The viewing angles are chosen to be about $16^{\circ}$ to the midplane and about $20^{\circ}$ to the major radius, with the antenna position of $20 \mathrm{~cm}$ below the midplane. The shaded region is the auxiliary plane-stratified plasma slab geometry used in the numerical full-wave solution.

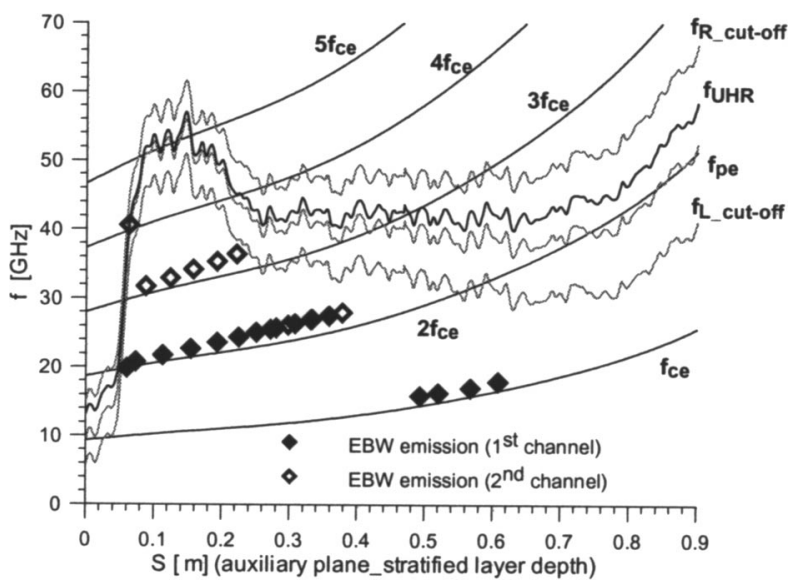

FIG. 2. Cutoffs and resonances in MAST. $S$ is the distance perpendicular to the separatrix from the spot position. EBW is emitted in the neighborhood of the position where $\left(\omega-n \omega_{c e}\right) /\left(k_{\|} \nu_{T}\right) \sim 3$. $[S=0.02 \mathrm{~m}$ at the separatrix because of the scrape-off layer (SOL) thickness.]

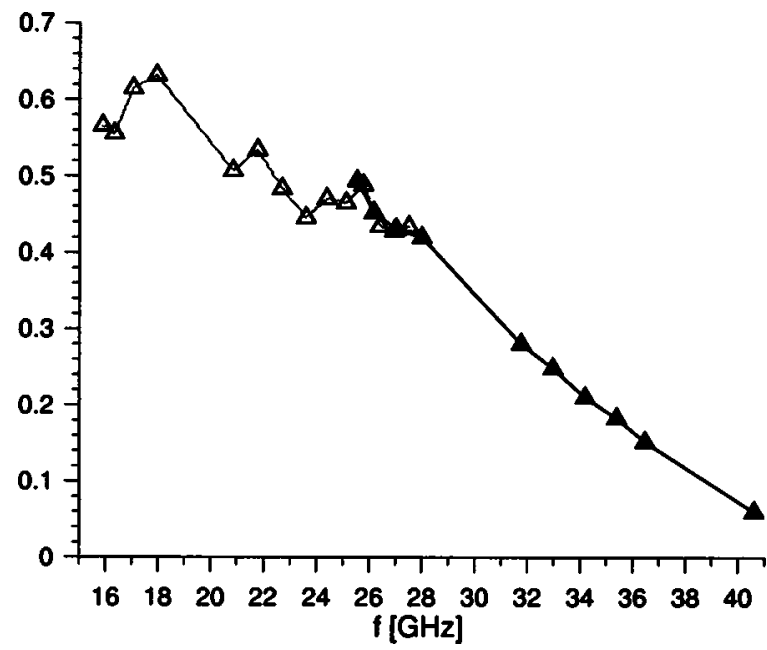

FIG. 3. The relative intensity of the linearly polarized component of the oblique $\mathrm{O}$ mode emitted at the plasma edge due to the $\mathrm{X}-\mathrm{O}$ mode conversion of the unity power $\mathrm{X}$ mode. The evanescent region increases with frequency, resulting in the conversion factor $C_{\mathrm{EBW}-\mathrm{X}-\mathrm{O}}$ decrease.

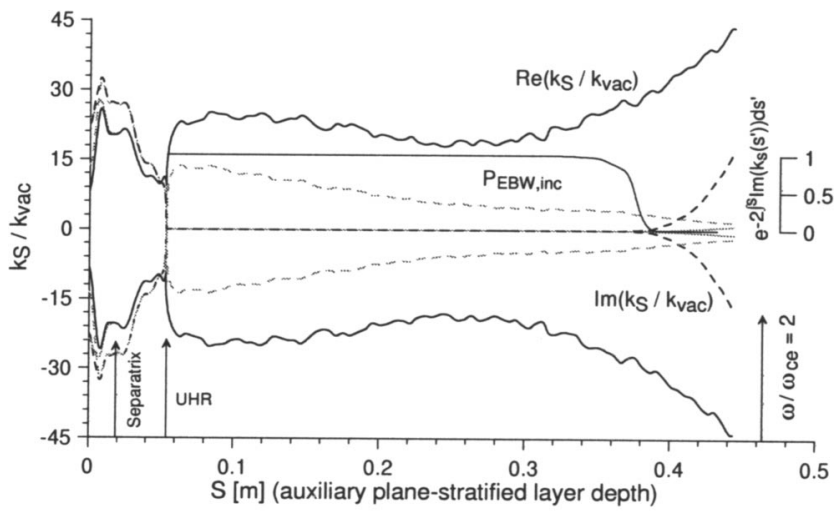

FIG. 4. The solution of the EBW dispersion relation $D(K, \omega)=0$ at $f$ $=27.98 \mathrm{GHz}$ for shot \#4958 at $t=120 \mathrm{~ms}$. $k_{s}$ is the wave number component parallel to the density gradient $\left(k_{\perp}=\sqrt{k_{S}^{2}+k_{\mathrm{pol}}^{2}}\right)$. At the plasma boundary, $k_{\|} / k_{\mathrm{vac}}=-0.66, k_{\mathrm{pol}} / k_{\mathrm{vac}}=-0.038$. The separatrix is at $S=0.02 \mathrm{~m}$. The electrostatic EBW propagates from UHR $(S=0.054 \mathrm{~m})$ until it is fully absorbed at $S=0.38 \mathrm{~m}$ (where the temperature is $0.99 \mathrm{keV}$ ).

$$
\begin{aligned}
D(\mathbf{k}, \omega)= & +\frac{2 \omega_{0}}{k^{2} \nu_{T}^{2}}\left[1+\frac{\omega}{\left|k_{\|}\right| \nu_{T}}\right. \\
& \left.\times \sum_{n=-\infty}^{\infty} Z\left(\frac{\omega-n \omega_{c e}}{\left|k_{\|}\right| \nu_{T}}\right) e^{-z_{L}} I_{n}\left(z_{L}\right)\right]=0,
\end{aligned}
$$

where $Z$ is the plasma dispersion function, $z_{L}=0.5$ $\left(k_{\perp} \nu_{T} / \omega_{c e}\right)^{2}, \nu_{T}=2 T_{e} / m_{e}$ is the electron thermal speed, $I_{n}$ is the modified Bessel function, $\omega_{0}(R, Z)$ is the local plasma frequency, and $\omega_{c e}(R, Z)$ is the local electron cyclotron frequency. $k_{\|}$and $k_{\perp}$ are the wave vector components parallel and perpendicular, respectively, to the local $\mathbf{B}$ field. In Figs. 4 and 5 we plot the complex wave number $k_{S}$ solution to $D(\mathbf{k}, \omega)=0$ at 27.98 and at $20.84 \mathrm{GHz}$, where $k_{S}$ is the component parallel to the density gradient.

\section{INTERPRETATION OF EMISSION SPECTRA [SHOT \#4958]}

The cutoff and resonance topology in shot \#4958 allows the EBW mode converted emission from EC harmonics up to

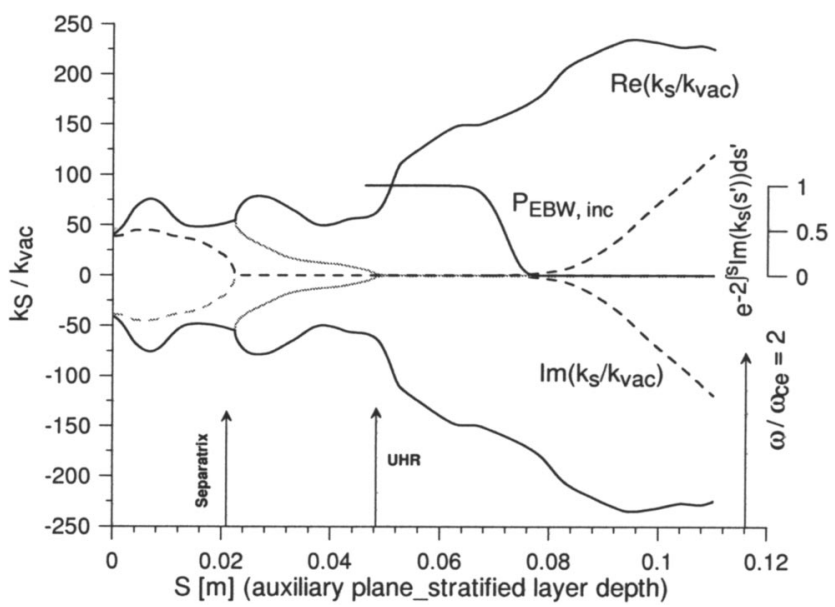

FIG. 5. The EBW wave number parallel to the density gradient $k_{s}$, for the same plasma conditions as in Fig. 4, but now at a lower frequency, $f$ $=20.84 \mathrm{GHz}$. The electrostatic EBW is now fully absorbed near the cold edge, $S=0.076 \mathrm{~m}$. 


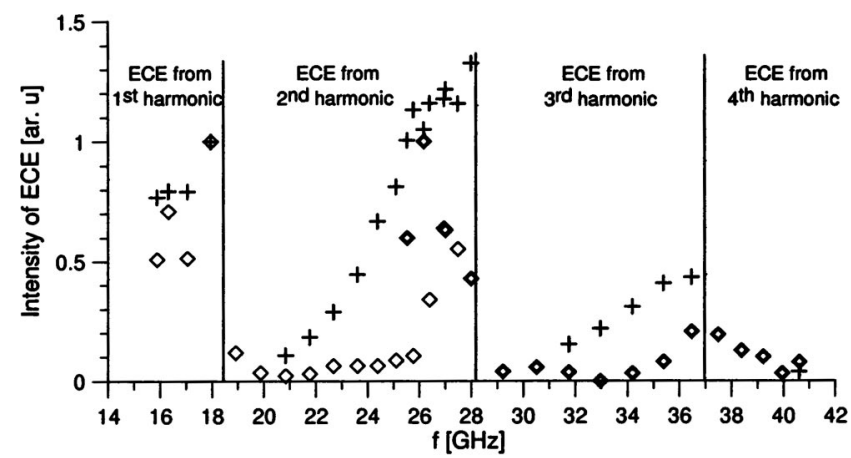

FIG. 6. ECE from MAST for $\# 4958$ at $t=120 \mathrm{~ms}$. Diamonds are experimental data for 32 frequencies in the two bands $(16-26$ and $26-40 \mathrm{GHz})$ and crosses are our theoretical estimates, using $f=17.94 \mathrm{GHz}$ as a reference frequency.

the fourth, as seen in Fig. 2. Thus, the plasma emission was reliably observed only in the first two frequency bands (16-26 and 26-40 GHz) of the frequency scanning EBW radiometer. ${ }^{3}$ These are plotted in Fig. 6 at time $t=120 \mathrm{~ms}$, when both the density and temperature were measured by Thomson scattering. Also shown is the theoretical ECE intensity, normalized to the experimental data at $f_{\text {ref }}$ $=17.94 \mathrm{GHz}$ :

$$
I_{\mathrm{ECE}}(f)=\frac{C_{\mathrm{EBW}-\mathrm{X}-\mathrm{O}}(f)}{C_{\mathrm{EBW}-\mathrm{X}-\mathrm{O}}\left(f_{\text {ref }}\right)} \frac{T_{\mathrm{EBWemission}}(f)}{T_{\mathrm{EBWemission}}\left(f_{\text {ref }}\right)} \frac{f^{2}}{f_{\text {ref }}^{2}} .
$$

We see that the frequency dependence of ECE splits into frequency bands that correspond to radiation from subsequent electron cyclotron harmonics. The positions of the bands, determined from the EFIT code, which gives correct values for magnetic fields at the plasma surface, coincide well with the intensity of the measured ECE. However, we see that the measured emission is substantially weaker for frequencies lying in the first two thirds of the interval (Fig. 6) between adjacent electron cyclotron harmonics. At present, we attribute this discrepancy during $\mathrm{H}$ mode to the modified magnetic field profile near the transport barrier region, caused by the bootstrap current, diamagnetic, and Pfirsch-Schluter currents that are not fully reconstructed by EFIT. The increased magnetic field could shift the resonance at the EC harmonics towards the plasma surface, and the eventual nonmonotonic magnetic field profile could block the radiation from the deeper part of the plasma. There is

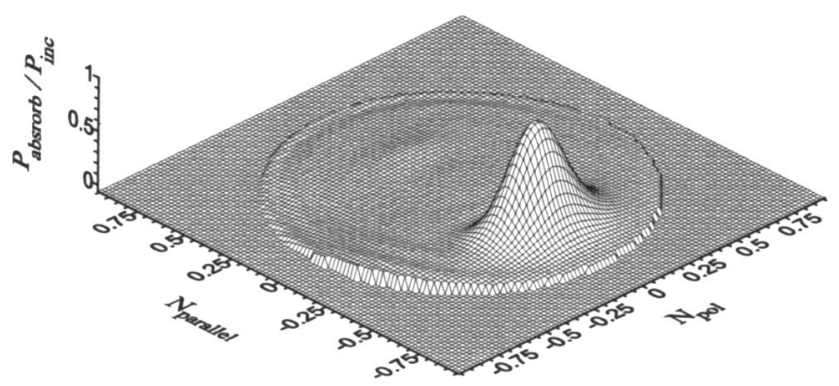

FIG. 8. The theoretical power absorption in a cold MAST plasma at $f$ $=58 \mathrm{GHz}$ (with weak collisions, $\nu / \omega=0.0005$ ) for a left-handed circularly polarized obliquely incident wave. Maximum absorption (96\%) occurs at $N_{\|}=-0.41, N_{\mathrm{pol}}=0$. Fifty percent absorption is reached for $N_{\|}= \pm 0.15$ $\left( \pm 8.5^{\circ}\right)$, and $N_{\mathrm{pol}}=-0.41 \pm 0.09\left( \pm 5^{\circ}\right)$.

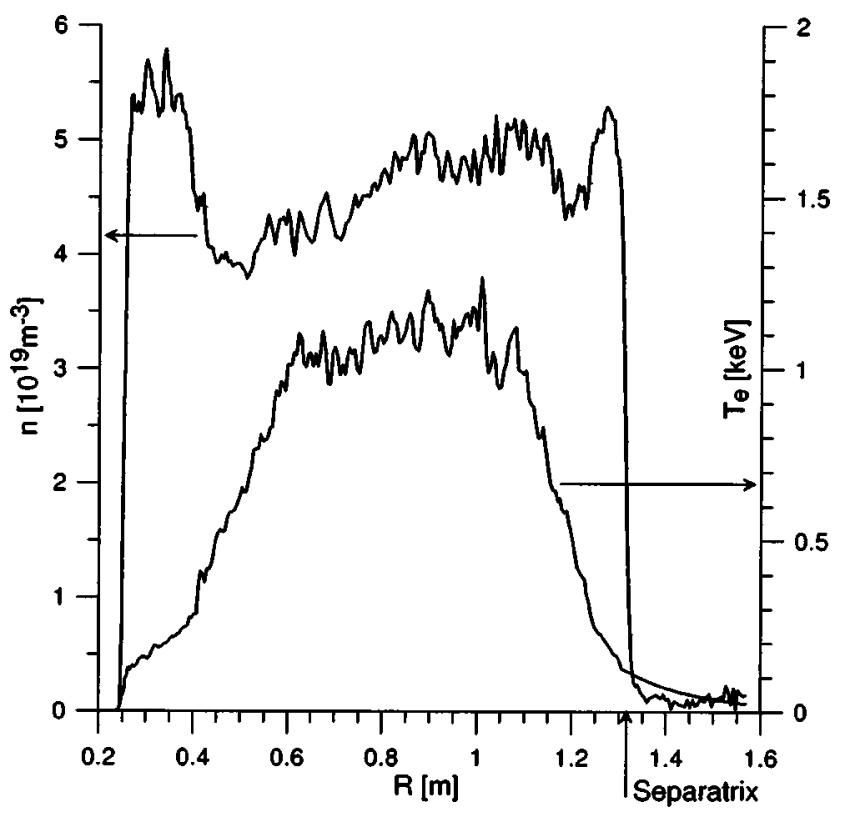

FIG. 7. Density (upper curve) and temperature (lower curve) profiles from high spatial resolution Thomson scattering measurement for shot \#6132 at $t=220 \mathrm{~ms}$

also an important difference in the dispersion properties of the long wavelength EBW with frequency directly below the electron cyclotron harmonic (e.g., for $f=17.94 \mathrm{GHz}$, we have at UHR, $\left.k_{S} / k_{\mathrm{vac}} \sim 3\right)$ and the short wavelength EBW above it (e.g., for $f=20.84 \mathrm{GHz}$, we have at UHR, $k_{S} / k_{\text {vac }}$ $\sim 63$ ). Together with the nonmonotonic profile of Debye shielding radius near the transport barrier, the short wavelength EBW with frequency slightly above harmonics has $k_{\perp} \lambda_{D} \approx 1$, even at the UHR, probably resulting in its strong damping. Further, the possible increase in $k_{\|}$(seen in the EBW ray-tracing analysis of Forest $^{6}$ ) could shift the absorption-emission region to substantially lower $T_{e}$.

\section{EBW AT $60 \mathrm{GHz}$}

In the near future, plasma heating and current drive will be investigated on MAST at $60 \mathrm{GHz}$. For this, one will re-

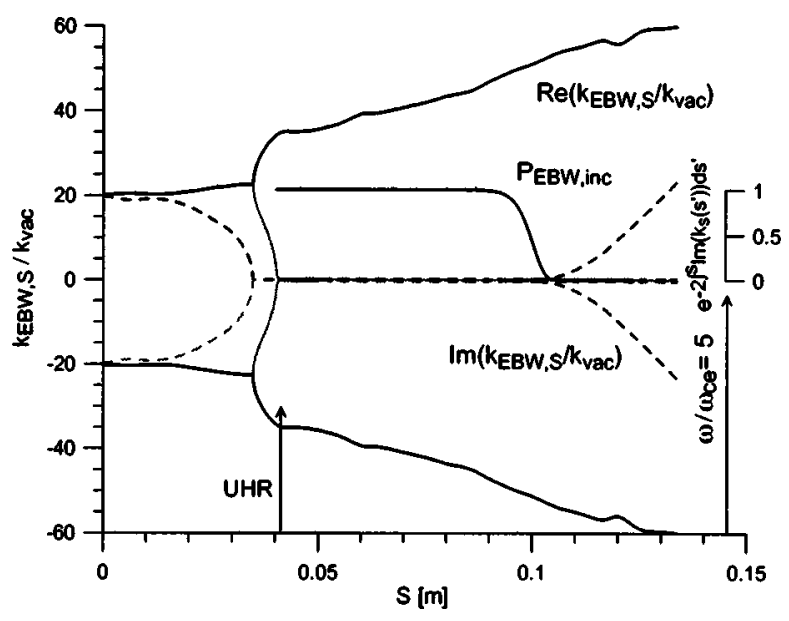

FIG. 9. The EBW wave number parallel to the density gradient $k_{s}$, for the steep edge density profiles in MAST, \#6132 (Fig. 7). The incident frequency is $f=58 \mathrm{GHz}$, and at the plasma edge $k_{\|} / k_{\mathrm{vac}}=-0.39, k_{\mathrm{pol}} / k_{\mathrm{vac}}=-0.01$. The electrostatic EBW is fully absorbed near the cold edge, $S=0.1 \mathrm{~m}$. 
quire high densities in a well-developed $\mathrm{H}$ mode. We thus investigate MAST discharge \#6132, which exhibits steep edge density gradients (Fig. 7) which will enable efficient conversion (the $\mathrm{O}-\mathrm{X}$-EBW) of a rather broad angular beam of incident circularly polarized $\mathrm{O}$ mode at oblique incidence. For a properly chosen incidence angle, the evanescent layer between the plasma resonance and the L cutoff of the $\mathrm{X}$ mode disappears and the $\mathrm{O}$ mode is fully converted to the $\mathrm{X}$ mode, which in turn is mode converted to EBW at the UHR. In Fig. 8 we show the absorbed power in a cold collisional full-wave model $^{7}$ of the MAST plasma. In the warm plasma model, this power will be converted to EBW.

The propagation of EBW in the warm plasma slab model is studied using the electrostatic dispersion relation [Eq. (1)] for $f=58 \mathrm{GHz}$ and plasma parameters for shot \#6132. The emitting antenna is assumed to be in the same orientation as before. In the envisaged experiment there will be an array of $7 \times 3$ independently steerable mirrors for the seven incident beams. From Fig. 9 one sees that the high frequency wave penetrates only to $S=0.1 \mathrm{~m}$. For such propagation, raytracing effects should be minimal. Higher plasma densities will improve the current drive somewhat.

\section{ACKNOWLEDGMENTS}

The work was partly supported by the Czech Grant Agency and U.S. DOE. The MAST program is jointly funded by the UK Department of Trade \& Industry and EURATOM.

\footnotetext{
${ }^{1}$ J. Preinhaelter and V. Kopecky, J. Plasma Phys. 10, 1 (1973).

${ }^{2}$ H. P. Laqua et al., 29th EPS, Montreux, 2002, paper O4.09 [Plasma Phys. Controlled Fusion 41, A273 (1999)].

${ }^{3}$ V. Shevchenko, 27th EPS, Budapest, 2000, paper P3.120.

${ }^{4}$ P. Pavlo, L. Krlin, and Z. Tluchor, Nucl. Fusion 31, 711 (1991).

${ }^{5}$ T. H. Stix, Waves in Plasmas (AIP, Melville, New York, 1992).

${ }^{6}$ C. B. Forest et al., Phys. Plasmas 7, 1352 (2000).

${ }^{7}$ J. Preinhaelter, V Shevchenko, M. A. Irzak, L. Vahala, and G. Vahala, Report UKAEA FUS 444, November (2000).
} 